\title{
Image-Based Computational Evaluation of the Competing Effect of Atrial Wall Thickness and Fibrosis on Re-Entrant Drivers for Atrial Arrhythmias
}

\author{
Aditi Roy, Marta Varela, Oleg Aslanidi \\ Kings College London, United Kingdom
}

\begin{abstract}
Catheter ablation is a common treatment for atrial fibrillation $(A F)$, but long-term patient outcomes remain suboptimal. Knowledge of the location of re-entrant drivers (RDs) sustaining AF can help optimize ablation strategies for individual patients. Increasing evidence suggest that both fibrosis and atrial wall thickness (AWT) can influence the RDs dynamics. This study aims to analyse the role of fibrotic patches and AWT in determining $R D$ sites in human right (RA) and left (LA) atrial models.

Atrial geometries and fibrosis distribution were obtained from 2 healthy volunteers and $2 \mathrm{AF}$ patients using $M R$ imaging. These 4 subject-specific geometries were integrated into $3 D$ atrial models with the Fenton-Karma model adopted to reproduce atrial electrophysiology.

In the RA model without fibrosis, RDs anchored to the crista terminalis $(C T)$ if initiated near a prominent $A W T$ gradient between this bundle and surrounding $R A$. In the presence of fibrosis, RDs either pinned between the CT and fibrotic patch or anchored to the latter, depending on the distance from their initiation site to the CT. In the LA model without fibrosis, RDs drifted towards the thinner pulmonary veins. However, with fibrotic patches added, $R D s$ either anchored around them or broke down into multiple wavelets. These findings can help identify $R D$ locations from imaging data and guide ablation therapy.
\end{abstract}

1. Introduction

Atrial fibrillation (AF) is the most commonly sustained cardiac arrhythmia. It is characterized by complex spatiotemporal organization [1] and is associated with increased risk of heart failure and stroke [2]. Catheter ablation is a well-established treatment option for $\mathrm{AF}$ which allows for specific destruction of arrhythmogenic tissue [3]. The efficiency of ablation procedures could be greatly improved if there were reliable methods for the accurate identification of the regions that harbour the reentrant drivers (RD) sustaining AF. Ablation procedures for paroxysmal $\mathrm{AF}$ have been successful by isolation of the pulmonary veins $(\mathrm{PV})$ which are the primary location of RDs in the left atrium [4]. However, the progression of AF results in ionic and structural remodelling of the atrial substrates, making RD locations harder to predict [5]. In such cases, identifying atrial RD locations requires a better understanding of the interplay between structural and functional mechanisms of AF in individual patients.

Recent experimental evidence from animal models have suggested that RDs can anchor to regions with large AWT [6], but studies to confirm this observation in realistic human atrial geometries are lacking. In addition to AWT, increasing evidence suggest that atrial fibrotic regions may play an important role in RD initiation and anchoring by providing regions of slow and/or irregular conduction [7].

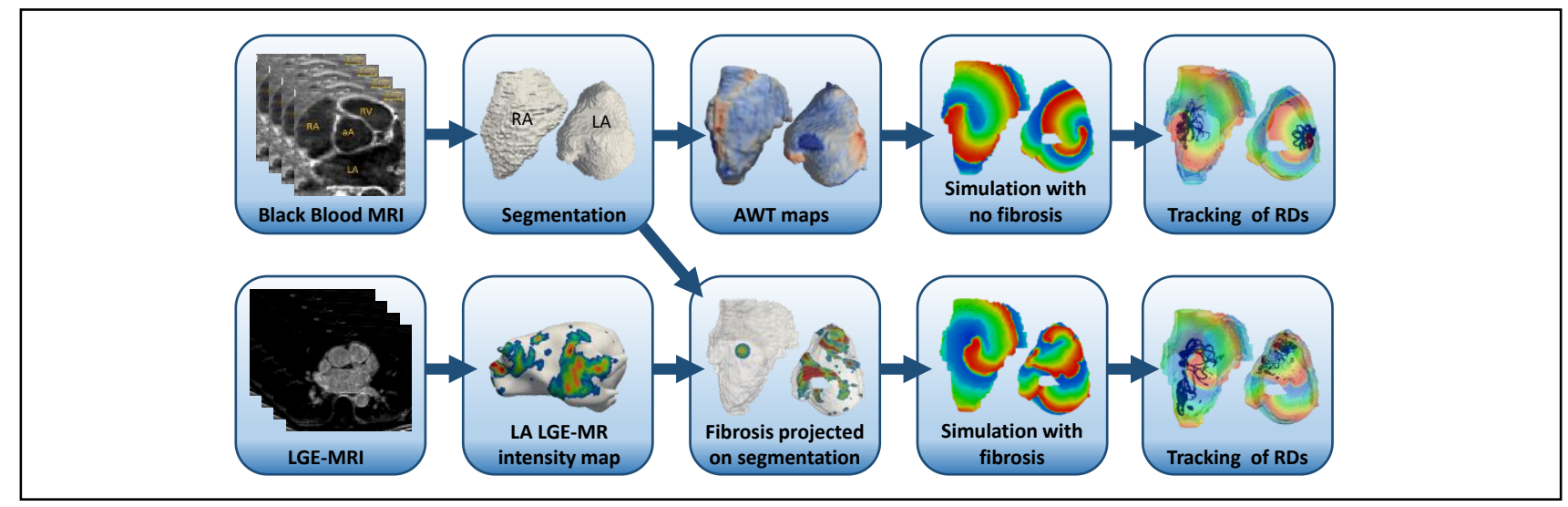

Figure 1: Workflow for the extraction of atrial geometries (top row) and fibrosis (bottom row) from MRI data and registering and then projecting them to evaluate the effect of fibrotic patches and AWT gradients on the RDs dynamics. 
A better understanding of the role of structural factors, such as AWT and fibrosis, may help determine optimal ablation sites. In this study, we aim to investigate the role of AWT and fibrotic patches in determining anchoring of RDs using computational modelling performed on 3D atrial geometries and fibrosis obtained from subjectspecific magnetic resonance imaging (MRI) data.

\section{Methods}

The modelling workflow consisted of subject-specific extracting atrial geometries and fibrosis distributions from MRI, integrating them into 3D human atrial models and implementing $\mathrm{AF}$ simulations, with and without the presence of AWT gradients and fibrosis. A schematic of this workflow is shown in Figure 1.

\subsection{AWT Measurements}

Two healthy volunteers and two AF patients were scanned using a novel black-blood PSIR MRI protocol (1.5T, cardiac and respiratory gated, $1.4 \times 1.4 \times 1.4 \mathrm{~mm}$ ). The right (RA) and left atrial (LA) geometries of healthy volunteers and patients, respectively, were obtained by manual segmentation using ITK Snap. The AWT maps for all geometries were computed using previously published methods [8]. This involved the generation of epicardial and endocardial meshes from the segmentations and the computation of the AWT maps by averaging the distance between nearest nodes on corresponding meshes.

\subsection{Fibrosis Imaging}

The two AF patients also underwent LGE MRI acquisition in the same scanning session (1.5T, cardiac and respiratory gating, $1.3 \times 1.3 \times 2 \mathrm{~mm}$ ) for detection of fibrosis in the LA. Due to constraints in scan time, LGE data was not acquired in the RA. Segmentation of fibrotic tissue was performed by analysing the image intensity ratio (IIR), computed by dividing individual voxel intensities by the mean blood pool intensity. IIR values $>1.08$ were classified as interstitial fibrosis and $>1.32$ as dense fibrosis. The IIR threshold of 1.08 was obtained as an average of the previously proposed values of 1.2 [9] and 0.97 [10]. Interstitial to dense fibrosis was modelled by labelling segmented fibrotic tissue from 1 to 5 based on LGE MRI intensity. These fibrotic regions were then registered with and projected onto the LA geometries for the same patients. In the RA, due to lack of fibrosis data we added a 3D spherical fibrotic patch of radius $4.5 \mathrm{~mm}$, with labels 5 to 1 from the centre to outside as shown in Figure 2A, B.

\subsection{D Atrial Simulations}

The 3D atrial model integrated subject-specific atrial geometries with the Fenton Karma electrophysiology model modified to accurately described the restitution properties of remodelled atrial cells [11]. The simulations were performed by solving the monodomain equation (1) with no flux boundary conditions, separately on the LA and RA, using forward Euler finite differences schemes with a

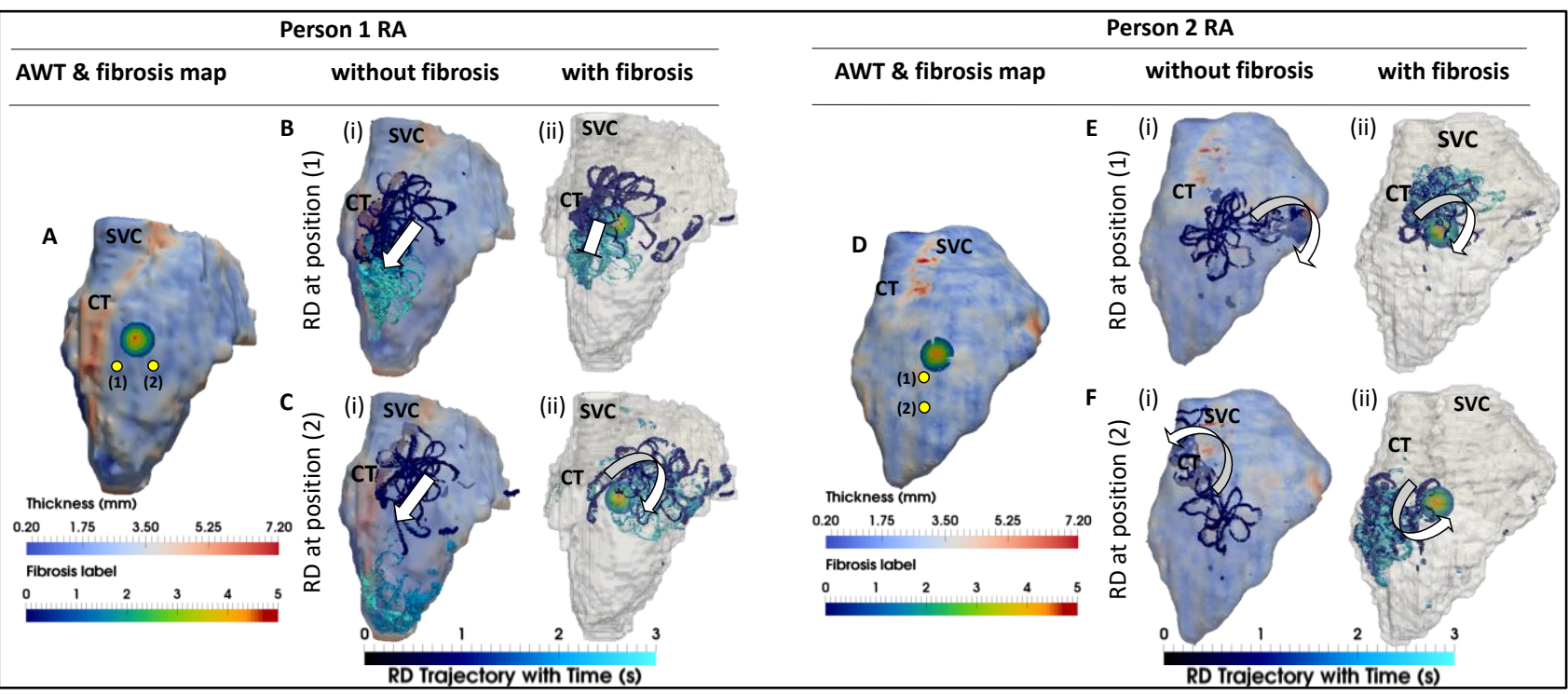

Figure 2: The RDs trajectories (blue) in the RA, obtained by AF simulation at two locations (yellow dots), without and with the presence of fibrosis have been overlaid on the AWT map and fibrosis distribution (A \& D), respectively for person 1 (left) and 2 (right). In person 1, without fibrosis, RDs initiated at both positions 1 and 2 drifted towards the CT, with a large AWT gradient shown in B (i) and C (i), respectively. However, with fibrosis, RDs got pinned when initiated at position 1 (B (ii)), located between the CT and fibrotic patch and fully anchored to the latter for position 2 (C (ii)), located further away from CT. In person 2, without fibrosis, RDs initiated at both positions (E (i) \& F (i)), neither drifted towards the thinner CT nor showed anchoring. Instead, with fibrosis, RDs initiated at both positions (E (ii) \& F (ii)) anchored to and drifted around the fibrotic patch. CT: Crista terminalis, SVC: Superior vena cava. 
temporal and spatial resolution of $0.005 \mathrm{~ms}$ and $0.3 \mathrm{~mm}$.

$$
\frac{\partial V}{\partial t}=\nabla \cdot(\boldsymbol{D} \nabla V)-\frac{I_{i o n}}{C_{m}}
$$

Here $\nabla$ is a spatial gradient operator, $V(\mathrm{mV})$ is the membrane potential and $t$ is time (ms). $\boldsymbol{D}$ is the tensor with diffusion coefficients $\left(\mathrm{mm}^{2} \mathrm{~ms}^{-1}\right)$ that characterises the spread of membrane potential within the tissue, $C_{m}(\mathrm{pF})$ is the membrane capacitance, and $I_{\text {ion }}$ is the total membrane ionic current (pA). Our model was isotropic and the diffusion tensor was replaced by a scalar diffusion coefficient $D=0.1 \mathrm{~mm}^{2} \mathrm{~ms}^{-1}$, carefully chosen to match an atrial conduction velocity of $0.65 \mathrm{~m} \mathrm{~s}^{-1}$ typical of AF. The effect of fibrosis on slowing atrial conduction [7] was modelled by a progressive decrease in the diffusive coupling by reducing $D$ within segmented fibrotic tissue by $17 \%$ to $83 \%$ for tissues labelled 1 to 5 , respectively.

RDs were initiated at different locations within the atrial geometry using a cross-field protocol, and their phase singularity points were tracked. Each tracked RD trajectory was then overlaid separately on AWT and fibrosis maps of individual patient's atrial geometry. Each simulation was performed with and without the presence of fibrosis for a duration of $3 \mathrm{~s}$ in the RA and $5 \mathrm{~s}$ in the LA.

\section{Results}

\subsection{Simulations in the RA}

The AWT map computed for one of the subjects (Figure 2A) showed a prominent thickness gradient at the crista terminalis (CT), $3.46 \pm 0.86 \mathrm{~mm}$ compared to the surrounding RA wall, $2.09 \pm 0.38 \mathrm{~mm}$. In this subject, 6 out of 9 of the initiated RDs drifted towards the CT, either anchoring to it (Figure 2B (i), C (i)) or drifting over it to the opposite side of the geometry. However, in the RA geometry from another subject (Figure 2D), the CT was not easily distinguishable from the remaining RA wall. Consequently, the RDs neither drifted towards the CT, nor showed anchoring at any location (Figure 2E (i), F (i)).

When these simulations were repeated in the presence of the synthetic fibrotic patch, the RD trajectories were affected in both subjects, as shown in (ii) columns of Figure 2. For the RA with a prominent CT (Figure 2A), the RDs initiated closer to the fibrotic patch either got pinned in a region between the CT and the patch (Figure 2B (ii)), or anchored to the outer boundary of the latter (Figure 2C (ii)), depending on the distance of their initiation site to either structures. In the RA with no thickness gradient at the CT (Figure 2D), all the RDs anchored to the fibrotic patch (Figure 2E (ii) and F (ii)).

\subsection{Simulations in the LA}

The AWT maps computed for one of the patient's LA geometry (Figure 3A) showed a high AWT at the LA roof $(4.77 \pm 0.60 \mathrm{~mm})$ compared to rest of the atrial wall $(2.96$ $\pm 0.31 \mathrm{~mm})$. The RDs were seen to drift towards and along the LA roof (Figure 3C (i)), when initiated in its proximity, and the ones initiated further away drifted towards the PV (Figure 3B (i)). The AWT map for another patient's LA (Figure 3D) was characterised by roughly uniform thickness of $2.53 \pm 1.20 \mathrm{~mm}$. The RDs initiated in this geometry drifted either towards the slightly thinner PVs (Figure 3E (i)) or the mitral valve (Figure 3F (i)).

The LGE MRI intensity-based segmentations yielded different fibrosis distributions in the $2 \mathrm{AF}$ patients, as shown in Figure 3A, D. Both patient's LAs had fibrotic tissue in regions surrounding the PVs, with higher degree
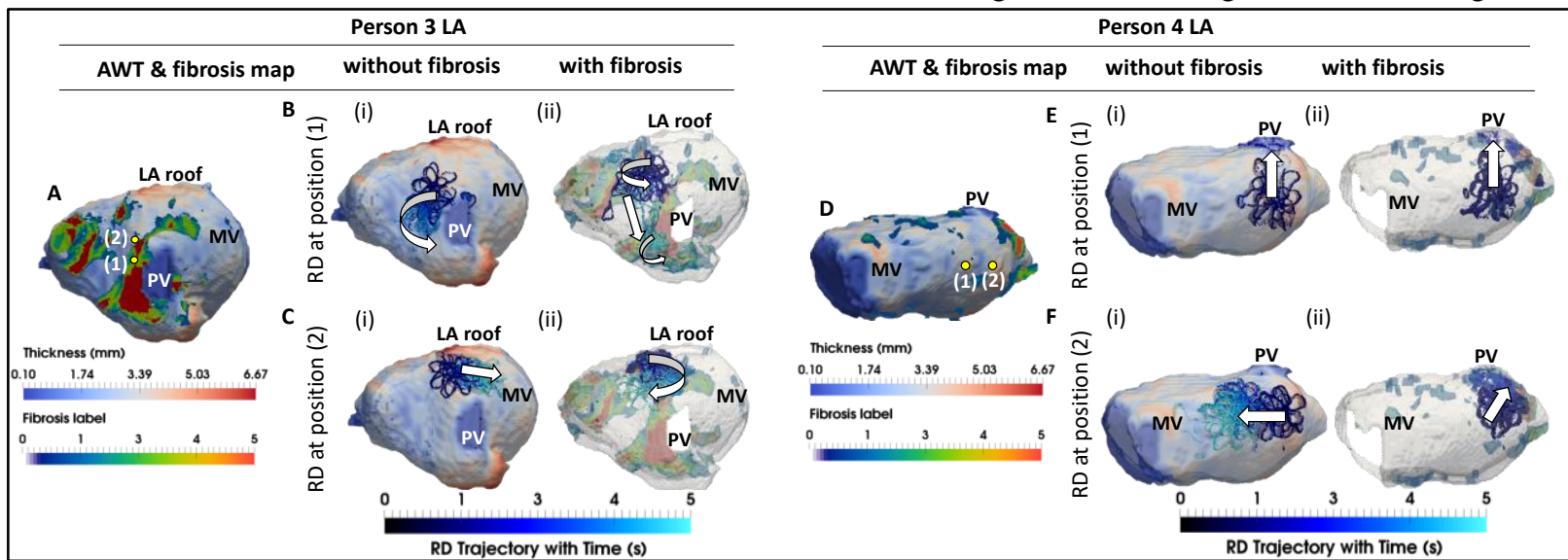

Figure 3: The RDs trajectories (blue) in the LA, obtained by AF simulation at two locations (yellow dots), without and with the presence of fibrosis have been overlaid on the AWT map and fibrosis distribution (A \& D), respectively for person 3 (left) and 4 (right). In person 3, without fibrosis, RDs initiated at position 1 and 2 were seen to drift towards the PVs (B (i)) and LA roof (C (i)), respectively. However, with fibrosis, RDs destabilized from its initial location to a secondary location (B (ii)) for position 1 and anchored to the fibrotic regions (C (ii)) for position 2. In person 4, without fibrosis, RDs initiated at positions 1 and 2 drifted towards the PVs (E (i)) and MV (F (i)), respectively. However, with fibrosis, RDs continued to drift towards the PVs (E (ii)) for position 1, but anchored to the fibrotic region (F (ii)) for position 2. PV: Pulmonary vein, MV: Mitral valve. 
of fibrosis in one patient (Figure 3A) compared to the other (Figure 3D). In the LA model, simulations repeated with the presence of fibrosis showed variable RD behaviours. In some cases, there was stable anchoring to the border zones of the fibrotic tissue (Figure 3C (ii), F (ii)), while in others there was breakdown of the primary RD into multiple RDs in fibrotic regions. In addition, the displacement of the RD from its initial anchoring site to a secondary location surrounding a fibrotic patch was observed (Figure 3B (ii)).

\section{Discussion}

The results obtained from the RA and LA model simulations suggest that the RD dynamics are highly dependent on the structural makeup of individual patient geometries. In the RAs, majority of atria tachycardia have been recorded along the long axis of the CT [12]. Our results suggest that RDs anchor to the $\mathrm{CT}$ in the absence of fibrosis, in patients with a large gradient in AWT at the CT compared to the surrounding RA. Hence, RDs are more likely to be found along the CT for these patients compared to ones that lack a relatively thick CT.

The interaction between fibrosis and heterogeneity in AWT played a major in determining RDs behavior in the RA. Using a synthetic patch of fibrotic tissue located near the CT, we showed the pinning of RDs at locations where the influence of the fibrotic tissue was balanced by the competing influence of the AWT gradient of the CT. RDs fully anchored to the fibrotic patch only when RDs were initiated further away from the CT with large AWT gradients. Such behavior suggests that the location of RDs is dependent on the interaction between these two structural components. Therefore, patient-specific imaging of AWT and fibrosis in the RA could be helpful when planning ablation strategies. This hypothesis, initially drawn from observations in the RA, was then tested in simulations with the LA using realistic fibrosis data.

In the LA in the absence of fibrosis, RDs most often localized in the regions surrounding the thinner PVs. However, with fibrotic tissue present, RDs either anchored around them or broke down into multiple wavelets. These results contribute to the understanding of why PV ablations are optimal in paroxysmal AF patients with a low fibrotic burden. Therefore, assessment of fibrosis in AF patients may assist in the prediction of sites where RDs anchor.

In summary, the CT with high AWT gradients can be the primary anchoring location for RDs in the RA. However, the RD locations in the LA were more sensitive to presence of fibrotic tissue. Hence, noninvasive assessment of AWT and fibrosis may contribute towards AF patient stratification and guidance of ablation therapy.

\section{Acknowledgements}

This research was funded by the National Institute for
Health Research (NIHR) Biomedical Research Centre, British Heart Foundation (PG/15/8/31138), King's College London and Imperial College London EPSRC Centre for Doctoral Training in Medical Imaging (EP/L015226/1).

\section{References}

[1] Zipes DP, Jalife J. Cardiac Electrophysiology: From Cell to Bedside. Elsevier Inc. 2014.

[2] Chugh SS, Havmoeller R, Narayanan K, Singh D, Rienstra M, Benjamin EJ, et al. Worldwide Epidemiology of Atrial Fibrillation. Circulation. 2014;129:837-847.

[3] Nattel S. New ideas about atrial fibrillation 50 years on. Nature. 2002;415:219-26.

[4] Oral H, Knight BP, Tada H, Özaydın M, Chugh A, Hassan S, et al. Pulmonary Vein Isolation for Paroxysmal and Persistent Atrial Fibrillation. Circulation. 2002;105:1077-1081.

[5] Nattel S, Burstein B, Dobrev D. Atrial remodeling and atrial fibrillation: mechanisms and implications. Circ Arrhythm Electrophysiol. 2008;1:62-73.

[6] Brec J, Yamazaki M, Mironov S, Vaquero LM, Bandaru $\mathrm{K}$, Avula UMR, et al. Heterogeneous atrial wall thickness and stretch promote scroll waves anchoring during atrial fibrillation. 2012;94:48-57.

[7] Morgan R, Colman MA, Chubb H, Seemann G, Aslanidi OV. Slow conduction in the border zones of patchy fibrosis stabilizes the drivers for atrial fibrillation: Insights from multi-scale human atrial modeling. Front Physiol. 2016;7:1-15.

[8] Varela M, Morgan R, Theron A, Dillon-Murphy D, Chubb H, Whitaker J, et al. Novel MRI Technique Enables Non-Invasive Measurement of Atrial Wall Thickness. IEEE Trans Med Imaging. 2017; 36(8):16071614.

[9] Benito EM, Carlosena-remirez A, Guasch E, Perea RJ, Figueras $\mathrm{R}$, Borra $\mathrm{R}$, et al. Left atrial fibrosis quantification by late gadolinium-enhanced magnetic resonance : a new method to standardize the thresholds for reproducibility. 2016; pii: euw219.

[10] Khurram IM, Beinart R, Zipunnikov V, Dewire J, Yarmohammadi H, Sasaki T, et al. Magnetic resonance image intensity ratio, a normalized measure to enable interpatient comparability of left atrial fibrosis. Heart Rhythm. 2014;11:85-92.

[11] Goodman AM, Oliver RA, Henriquez CS, Wolf PD. A membrane model of electrically remodelled atrial myocardium derived from in vivo measurements. Europace. 2005;7(Suppl 2):S135-S145.

[12] Kalman JM, Olgin JE, Karch MR, Hamdan M, Lee RJ, Lesh MD. "Cristal tachycardias": Origin of right atrial tachycardias from the crista terminalis identified by intracardiac echocardiography. J Am Coll Cardiol. $1998 ; 31: 451-9$.

\section{Address for correspondence}

Miss Aditi Roy; Division of Imaging Sciences \& Biomedical Engineering, King's College London, St. Thomas' Hospital, London SE1 7EH, UK; Email: aditi.roy@kcl.ac.uk 